\title{
PGC-1 $\alpha$ Participates in the Protective Effect of Chronic Intermittent Hypobaric Hypoxia on Cardiomyocytes
}

\author{
Shuo Gua,b Hong Hua ${ }^{a} \quad$ Xinqi Guo ${ }^{a}$ Zhanfeng Jiac ${ }^{c}$ Yi Zhanga \\ Leonid N. Maslov ${ }^{d} \quad$ Xiangjian Zhang ${ }^{b}$ Huijie Ma,b \\ aDepartment of Physiology, Hebei Medical University, Shijiazhuang, ${ }^{b}$ Hebei Collaborative Innovation \\ Center for Cardio-Cerebrovascular Disease, Shijiazhuang, 'Department of Pharmacology, Hebei Medical \\ University, Shijiazhuang, China, dLaboratory of Experimental Cardiology, Federal State Budgetary \\ Scientific Institution, Research Institute for Cardiology, Tomsk, Russia
}

\section{Key Words}

Chronic intermittent hypobaric hypoxia - Cardiomyocytes - Calcium overload - PGC-1 $\alpha$ • Energy metabolism • Hypoxia/reoxygenation

\begin{abstract}
Background/Aims: Myocardial ischemia/reperfusion (I/R) or hypoxia/reoxygenation (H/R) injury is always characterized by $\mathrm{Ca}^{2+}$ overload, energy metabolism disorder and necrocytosis of cardiomyocytes. We showed previously that chronic intermittent hypobaric hypoxia $(\mathrm{ClHH})$ improves cardiac function during I/R through improving cardiac glucose metabolism. However, the underlying cellular and molecular mechanisms of $\mathrm{ClHH}$ treatment improving energy metabolism in cardiomyocytes are still unclear. In this study, we determined whether and how $\mathrm{ClHH}$ protects cardiomyocytes from $\mathrm{Ca}^{2+}$ overload and necrocytosis through energy regulating pathway. Methods: Adult male Sprague-Dawley rats were randomly divided into two groups: control $(\mathrm{CON})$ and $\mathrm{ClHH}$ group. $\mathrm{ClHH}$ rats received a hypobaric hypoxia simulating 5,000-m altitude for 28 days, 6 hours each day, in hypobaric chamber. Rat ventricular myocytes were obtained by enzymatic dissociation. The intracellular calcium concentration $\left(\left[\mathrm{Ca}^{2+}\right]_{i}\right)$ and $\mathrm{cTnl}$ protein expression were used to evaluate the degree of cardiomyocytes injury during and after H/R. The mRNA and protein expressions involved in cardiac energy metabolism were determined using quantitative PCR and Western blot techniques. PGC- $1 \alpha$ siRNA adenovirus transfection was used to knock down PGC-1 $\alpha$ gene expression of cardiomyocytes to determine the effect of PGC-1 $\alpha$ in the energy regulating pathway. Results: $\mathrm{H} / \mathrm{R}$ increased $\left[\mathrm{Ca}^{2+}\right]_{\mathrm{i}}$ and $\mathrm{cTnl}$ protein expression in cardiomyocytes. $\mathrm{ClHH}$ treatment decreased $\left[\mathrm{Ca}^{2+}\right]_{\mathrm{i}}(p<0.01)$ and $c T n l$ protein expression $(p<0.01)$ in cardiomyocytes after $\mathrm{H} / \mathrm{R}$. Both mRNA and protein expression of PGC- $1 \alpha$ increased after $\mathrm{ClHH}$ treatment, which was reversed by PGC- $1 \alpha$ siRNA adenovirus transfection. Furthermore, $\mathrm{ClHH}$ treatment increased the expression of HIF- $1 \alpha$, AMPK and p-AMPK in cardiomyocytes, and pretreatment with AMPK inhibitor dorsomorphin abolished the enhancement of PGC-1 $\alpha$ protein expression

\begin{tabular}{ll}
\hline Huijie Ma & Department of Physiology, Hebei Medical University \\
& Shijiazhuang (China) \\
& E-Mail lily564300@163.com
\end{tabular}
\end{abstract}

\section{KARGER}


in cardiomyocytes by $\mathrm{CIHH}(p<0.01)$. In addition, PGC- $1 \alpha$ knock down also abolished the increased protein level of GLUT4 $(p<0.01)$ and decreased the protein level of CPT-1b $(p<0.05)$ in cardiomyocytes by $\mathrm{ClHH}$ treatment. Conclusion: $\mathrm{ClHH}$ treatment could reduce the calcium overload and $H / R$ injury in cardiomyocytes by up-regulating the expression of PGC-1 $\alpha$ and regulating the energy metabolism of glucose and lipid. The HIF-1 $\alpha-A M P K$ signaling pathway might be involved in the process.

(C) 2018 The Author(s)

Published by S. Karger AG, Basel

\section{Introduction}

Ischemic myocardial disease and myocardial infarction are still the most common causes of morbidity and mortality globally [1]. Myocardial ischemia/reperfusion (I/R) or hypoxia/reoxygenation (H/R) injury is always characterized by $\mathrm{Ca}^{2+}$ overload, generation of ROS and energy metabolism disorder [2,3]. These massive alterations of $\mathrm{Ca}^{2+}$ activate varieties of systems, and all of which can contribute to cell damage and death following I/R or H/R [2]. Disturbed energy supply or altered energy substrate utilization has also been found to be closely correlated with impaired cardiac function, and increases susceptibility to I/R or H/R injury [4, 5]. Despite recent advances in pharmacological and device therapies, the molecular mechanism underlying ischemic or hypoxic heart diseases remains unclear.

Chronic intermittent hypobaric hypoxia $(\mathrm{CIHH})$, which is similar to ischemic preconditioning and high altitude hypoxia adaptation, confers cardiac protection against ischemia/reperfusion (I/R) injury in rats [6-9]. Enhancement of resistance against calcium overload is one of the striking mechanisms of CIHH. Studies have shown that CIHH protects hearts from I/R injury and reduces calcium overload by preserving normal $\mathrm{I}(\mathrm{Na} / \mathrm{Ca})$ and NCX1, RyRs and SERCA2 proteins, and opening of the K(ATP) channel and inhibiting of opening of the MPTP $[8,10,11]$. We showed previously that CIHH treatment ameliorates I/R injury through improving cardiac glucose metabolism by improving cardiac glucose metabolism [12], yet whether the peroxisome proliferator-activated receptor gamma coactivator1-alpha (PGC-1 $\alpha$ ), a key molecule that regulate energy metabolism, and its related pathway plays a role in the anti-calcium overload in cardiomyocytes of CIHH has not been well addressed. We hypothesize that PGC- $1 \alpha$ also maintains intracellular calcium homeostasis while regulating cardiomyocyte metabolism, and plays an important role in maintaining cardiomyocyte's function. Therefore, in this study, we determined the role of PGC- $1 \alpha$ in the anti-calcium overload effect of $\mathrm{CIHH}$ in cardiomyocytes and its underlying mechanisms.

\section{Materials and Methods}

\section{Drugs}

Collagenase II was purchased from Worthington Biochemical Corporation (Lakewood, NJ,USA). Rhod-2AM was purchased from Dojindo Molecular Technologies, Inc. (Tokyo, Japan). Dorsomorphin dihydrochloride was purchased from MedChem Express (Monmouth Junction, NJ, USA). NaCl, KCl, glucose, 2, 4-dinitrophenol (DNP), taurine, glibenclamide, and EGTA were all purchased from Sigma-Aldrich Co (St Louis, MO, USA).

\section{Animals and CIHH procedure}

All animal experiments were conducted in compliance with the Guide for the Care and Use of Laboratory Animals (Publication 85-23, revised 1996; National Institutes of Health, Bethesda, MD), and all techniques and procedures were reviewed and approved by Hebei Medical University Animal Care\&Use Committee. Adult male Sprague-Dawley (SD) rats (8-10 weeks old) obtained from the Experimental Animal Center of Hebei Medical University were randomly divided into two groups: Control (CON) and CIHH group. The CIHH rats were put into to a hypobaric chamber to receive hypobaric hypoxia treatment mimicking 5, 000-m altitude ( $\mathrm{PB}=404 \mathrm{mmHg}, \mathrm{PO}_{2}=84 \mathrm{mmHg}, 6$ hours per day) for 28 days. The control rats were housed in the 


\section{Cellular Physiology Cell Physiol Biochem 2018;50:1891-1902 \begin{tabular}{l|l|l} 
and Biochemistry Published online: 3 November 2018 & $\begin{array}{l}\text { ○ } 2018 \text { The Author(s). Published by S. Karger AG, Basel } \\
\text { www.karger.com/cpb }\end{array}$ \\
\hline
\end{tabular}}

Gu et al:: CIHH Protects Cardiomyocytes via PGC- $1 \alpha$

same environment as the $\mathrm{CIHH}$ rats but without $\mathrm{CIHH}$ treatment. Both $\mathrm{CIHH}$ and CON rats were housed at room temperature with a natural light/ dark (12 hours/12 hours) cycle.

\section{Isolation of cardiomyocytes}

Single cardiomyocytes were isolated by enzymatic dissociation as described previously [8]. Briefly, rats were anaesthetized by intraperitoneal injection of urethane $(1.25 \mathrm{~g} / \mathrm{kg})$ and heparin $(300 \mathrm{U} / \mathrm{kg})$. The rat heart was excised and retrogradely perfused via the aorta for 5 minutes on a Langendorff apparatus (Chengdu Instrument, Sichuan, China) at a flow rate of $6 \mathrm{ml} / \mathrm{min}$ with oxygenated $\mathrm{Ca}^{2+}$-free Tyrode's solution containing (in mmol/L): $\mathrm{NaCl} 135, \mathrm{KCl} 5.4, \mathrm{MgCl}_{2}$ 1.0, $\mathrm{NaH}_{2} \mathrm{PO}_{4}$ 0.33, glucose 5, and 4-(2-hydroxyethyl)1-piperazineethanesulfonic acid (HEPES) 10 (gassed with $100 \% \mathrm{O}_{2}, \mathrm{pH}$ adjusted to 7.4 with $\mathrm{NaOH}$ ). The heart was then perfused with $\mathrm{Ca}^{2+}$-free Tyrode's solution containing collagenase II $(500 \mathrm{mg} / \mathrm{L})$ and $\mathrm{CaCl}_{2}(50$ $\mu \mathrm{mol} / \mathrm{L}$ ) at $37^{\circ} \mathrm{C}$ for 15 minutes. Finally, the left ventricle was removed and cut into a small pieces in a Kraft Brühe (KB) solution containing (in mmol/L): $\mathrm{KOH} \mathrm{80,} \mathrm{KCl} 40, \mathrm{KH}_{2} \mathrm{PO}_{4} 25, \mathrm{MgSO}_{4} 3$, glutamic acid 50, taurine 20 , ethylene glycol tetraacetic acid 1, HEPES 10 , and glucose 10 (gassed with $100 \% \mathrm{O}_{2}$, pH adjusted to 7.2 with $\mathrm{KOH}$ ). Cardiomyocytes were harvested after filtration through $200 \mu \mathrm{m}$ pore size nylon mesh and stored in a $\mathrm{KB}$ solution at room temperature for at least 1 hour; then the concentration of $\mathrm{Ca}^{2+}$ in the $\mathrm{KB}$ solution was gradually restored to $1.8 \mathrm{mmol} / \mathrm{L}$ before the experiment.

\section{Culture of cardiomyocytes}

Cardiomyocytes were cultured with Medium 199 supplemented with 5\% FBS, 1\% PS, 1\% ITS, 5 mM Taurine, $10 \mathrm{mM}$ BDM, $0.1 \mathrm{mg} / \mathrm{ml}$ BSA. The medium was added to a 6-well plate and placed in an incubator (gassed with $5 \% \mathrm{CO}_{2}, 37^{\circ} \mathrm{C}$ ) for 10 minutes. Then cardiomyocytes were seeded in 6-well plates at a cell number of 2-3 $\times 10^{5} /$ well and incubated in the incubator. Only rod-shaped cardiomyocytes with clear sarcolemma, but without spontaneous contraction in 5 minutes were used in the whole experiment.

\section{$P G C-1 \alpha$ siRNA adenovirus transfection}

The PGC-1 $\alpha$ siRNA adenovirus tagged with GFP fluorescence was constructed by Shanghai Han Heng Biotechnology Company. The cardiomyocytes were maintained in Medium 199 for 1 hour. The medium in the well was changed to the medium containing the PGC- $1 \alpha$ siRNA adenovirus (multiplicity of infection, MOI $=100$ ) and the cells were transfected in the incubator for 2 hours. Then we changed back to normal medium and cardiomyocytes were incubated in the incubator for additional 48 hours. The corresponding control group of cardiomyocytes was transfected with the control adenovirus without PGC-1 $\alpha$ siRNA using the same cell culture method and environment.

\section{Measurement of $\left[\mathrm{Ca}^{2+}\right]_{i}$}

Cardiomyocytes were incubated with intracellular calcium fluorescence indicator acetoxymethyl derivative (Rhod-2AM) solution containing $0.04 \%$ pluronic F-127 (the final concentration of Rhod-2AM is $20 \mu \mathrm{mol} / \mathrm{L}$ ) at $37^{\circ} \mathrm{C}$ for 30 minutes in dark. The cardiomyocytes were washed three times with PBS buffer to remove the extracellular Rhod-2AM. The cuvette was washed twice in PBS solution according to the test requirements and then mounted on a microscope stage (IRBE, Leica, Germany). Cardiomyocytes were added to perfusion chamber, until the cells adherent. Rhod-2AM fluorescence was excited at $557 \mathrm{~nm}$ from a high-power argon ion laser, and the fluorescent signal was detected with a confocal TCS-SP laser scanning system (Leica, Germany) equipped with an IRBE microscope (Leica, Germany). The change in $\left[\mathrm{Ca}^{2+}\right]_{\mathrm{i}}$ was represented by relative fluorescence intensity $\left(\left(\mathrm{FI}_{-}-\mathrm{FI}_{0}\right) / \mathrm{FI}_{0} \cdot 100 \%\right.$; $\mathrm{FI}_{0}$ : control; FI: damage). The $\left[\mathrm{Ca}^{2+}\right]_{\mathrm{i}}$ recording was performed at room temperature $\left(25^{\circ} \mathrm{C}\right)$. Fluorescence intensity was recorded and images were scanned and saved during each experiment according to the response of cardiomyocytes.

\section{RNA isolation and Quantitative Real-Time PCR}

Cardiomyocytes were collected and centrifuged at $1,000 \mathrm{rpm}, 4^{\circ} \mathrm{C}$ for 2 minutes. RNA was extracted from cardiomyocytes samples by using TRIzol reagent (Thermo, USA) method following manufactory's instruction. The integrity of total RNA was assessed using Nano Drop 2000 Spectrophotometer (Thermo, USA). Total RNA was reversed transcription using SYBR Select Master Mix Reverse Transcription Kit (Thermo, USA) according to the manufacturer's instructions. The cDNA was diluted (1:20) and used for real-time PCR. QuantiNova SYBR Green PCR Kit (QIAGEN, USA) were used to measure gene expression using specific primer 


\section{Cellular Physiology Cell Physiol Biochem 2018;50:1891-1902 \begin{tabular}{l|l|l|l|} 
and Bol: $10.1159 / 000494869$ & 2018 The Author(s). Published by S. Karger AG, Basel \\
www.karger.com/cpb
\end{tabular} \\ Published online: 3 November 2018 www.karger.com/cPb}

sets: PGC- $1 \alpha$ (forward primer: GAATGCAGCGCTCTTAGC; reverse primer: GCTTTTGCTGTTGACAAATG), and GAPDH (forward primer: GGACCAGGTTGTCTCCTGTG; reverse primer: ATTCGAGAGAAGGGAGGGCT, Invitrogen, USA). Dissociation curves were run following Real-Time PCR reactions to ensure the detection of the desired amplicon and excluded the presence of contaminating products. All reactions were performed in the ABI Prism 7000 Sequence Detection System (Applied Biosystems, USA). Gene expression was normalized to GAPDH and the data were analyzed using comparative $2^{-\Delta \Delta \mathrm{Ct}}$ method.

\section{Western blot analysis}

Cardiomyocytes were washed three times with PBS and centrifuged at $1,000 \mathrm{rpm}$ for 2 minutes at $4^{\circ} \mathrm{C}$. RIPA lysis buffer (Solarbio, China) containing protease cocktail and phostop was added to the cardiomyocyte precipitation and vortexed for 5 times and then incubated on ice for 20 minutes. After centrifugation at $12,000 \mathrm{rpm}$ and $4^{\circ} \mathrm{C}$ for 10 minutes, the supernatant was collected as total protein samples. The protein concentration was determined by bicinchoninic acid (BCA) assay. Fifty micrograms of total proteins were loaded and electrophoresed through polyacrylamide SDS gels and were then transferred by electroblotting onto polyvinylidene difluoride (PVDF) membranes. Membranes were blocked in 5\% (w/v) nonfat milk for 1 hour before incubated with antibodies of rabbit anti-PGC-1 $\alpha(1: 2,000$, Proteintech, China), rabbit antiHIF-1 $\alpha$ (1:300, Proteintech, China), rabbit anti-CPT-1b (1: 1, 000, Proteintech, China), rabbit anti-AMPK (1: 1, 000, Proteintech, China), rabbit anti-Phospho-AMPK (Thr 172) (1: 1, 000, Proteintech, China), rabbit antiGLUT4 (1:1, 000, Proteintech, China), rabbit anti-cTnI (1: 3, 000, Proteintech, China) and rabbit anti-GAPDH (1: 5, 000, Empitomics, USA) respectively at $4^{\circ} \mathrm{C}$ overnight. The PVDF membrane was washed 3 times and then incubated with horseradish peroxidase-conjugated anti-rabbit IgG $(1: 5,000$, Proteintech, China) for 1 hour at room temperature. The blots were detected using the ECL system (immobilion TM Western, Millipore) and analyzed by Quantity One Software (Bio-Rad, USA). Protein contents were normalized by GAPDH level on the same blot.

\section{Chemical hypoxia/reoxygenation of cardiomyocyte and experimental procedure}

After 48-hour's incubation, primary rat cardiomyocytes cultures were perfused with Tyrode's solution containing $1.8 \mathrm{mmol} / \mathrm{L} \mathrm{CaCl}_{2}$ (saturated with $95 \% \mathrm{O}_{2}$ and $5 \% \mathrm{CO}_{2}$ ) for 5 minutes in the perfusion chamber, and then exposed to Tyrode's solution containing 2, 4-dinitrophenol (DNP, $0.5 \mathrm{mmol} / \mathrm{L}$ ) to mimic myocardial chemical hypoxia for $20 \mathrm{~min}$, followed by reperfusion with the normal Tyrode's solution for 10 minutes (reoxygenation). Cells were collected by centrifugation $(1,000 \mathrm{rpm}, 2 \mathrm{~min}$ ) for subsequent experiments.

To determine whether AMPK participated in the effect of CIHH, AMPK (Adenosine 5'- monophosphate (AMP)-activated protein kinase) inhibitor dorsomorphin $(10 \mu \mathrm{mol} / \mathrm{L})$ was added to the culture medium of cardiomyocytes, and cardiomyocytes were incubated in the incubator for 15 hours [13]. Then the cardiomyocytes were incubated in normal medium until 48 hours from beginning of incubation.

\section{Data analysis}

Prism 6 (GraphPad, USA) was used for statistical analyses. All data were expressed as means \pm standard error of the mean (SEM). Parameter differences before and after H/R were analyzed using paired Student's t-test. Comparisons among groups were evaluated using one-way ANOVA followed by Tukey's post hoc test or two-way ANOVA followed by Sidak's post hoc test. Statistical significance was accepted when $p<0.05$.

\section{Results}

CIHH treatment alleviated calcium overload and $H / R$ injury of cardiomyocytes

The $\left[\mathrm{Ca}^{2+}\right]_{\mathrm{i}}$ in ventricular myocytes increased after 20 minutes of chemical hypoxia followed by 10 minutes of reoxygenation in both CON and CIHH myocytes. This increase of $\left[\mathrm{Ca}^{2+}\right]_{\mathrm{i}}$ was significantly less in the CIHH myocytes than that in the CON myocytes $(p<0.01$, Fig. 1A). Furthermore, cTnI, a myocardial injury marker protein, increased dramatically after $\mathrm{H} / \mathrm{R}$ in CON myocytes, but reduced in $\mathrm{CIHH}$ myocytes $(p<0.01$, Fig. $1 \mathrm{~B}, \mathrm{C})$. These data suggest that $\mathrm{CIHH}$ exerts a protective effect against $\mathrm{H} / \mathrm{R}$-induced calcium-overload and hypoxic injury in cardiomyocytes. 
Fig. 1. CIHH treatment alleviates calcium overload and hypoxia/ reoxygenation $(\mathrm{H} / \mathrm{R})$ induced injury of cardiomyocytes. A: Effect of $\mathrm{CIHH}$ on $\left[\mathrm{Ca}^{2+}\right]_{i}$ in cardiomyocytes undergoing $\mathrm{H} / \mathrm{R}$ represented by changes of relative fluorescent intensity in single isolated rat cardiomyocytes after $20 \mathrm{~min}$ chemical hypoxia and 10 min of reoxygenation. $\left[\mathrm{Ca}^{2+}\right]_{\mathrm{i}}$ : intracellular $\mathrm{Ca}^{2+}$ concentration. Representative Western blot images (B) and summary data (C) shows the effect of $\mathrm{CIHH}$ on cTnI protein expression in cardiomyocytes before and after H/R. CON, control group; CON H/R, control group with H/R; CIHH, CIHH group; CIHH H/R, CIHH group with $H / R$. Data are presented as mean $\pm S E M, n=10$ cells from 6 rats in each group. ${ }^{* *} \mathrm{p}<0.01$ vs. CON; ${ }^{*} \mathrm{p}<0.01$ vs. CIHH.
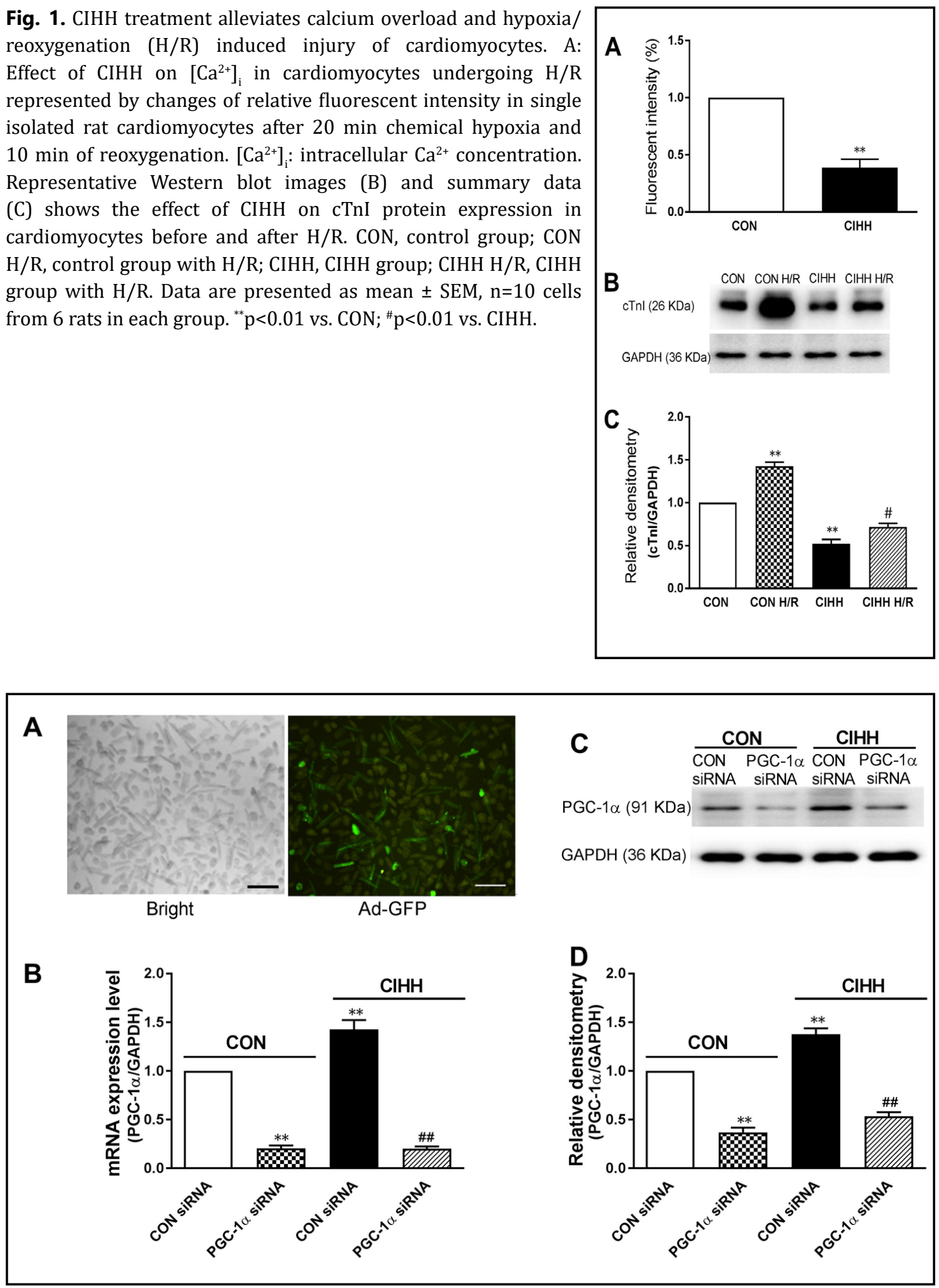

Fig. 2. PGC-1 $\alpha$ siRNA adenovirus transfection inhibits the protein and mRNA expression of PGC-1 $\alpha$. A: cardiomyocytes in bright field and epifluorescence images (Ad-GFP) 48 hours after transfected with PGC$1 \alpha$ siRNA eGFP adenovirus. Scale bar: $100 \mu \mathrm{m}$. B: PGC- $1 \alpha$ mRNA expression in the cardiomyocytes after transfected with control siRNA or PGC- $1 \alpha$ siRNA adenovirus. C: representative Western blot images and summary data show the changes of PGC- $1 \alpha$ protein level after transfected with control siRNA or PGC- $1 \alpha$ siRNA adenovirus. CON, control group; $\mathrm{CIHH}, \mathrm{CIHH}$ group. Data are presented as mean $\pm \mathrm{SEM}, \mathrm{n}=6$ rats in each group. ${ }^{* *} \mathrm{p}<0.01$ vs. CON siRNA of CON group; ${ }^{\# \#} \mathrm{p}<0.01$ vs. CON siRNA of CIHH group. 


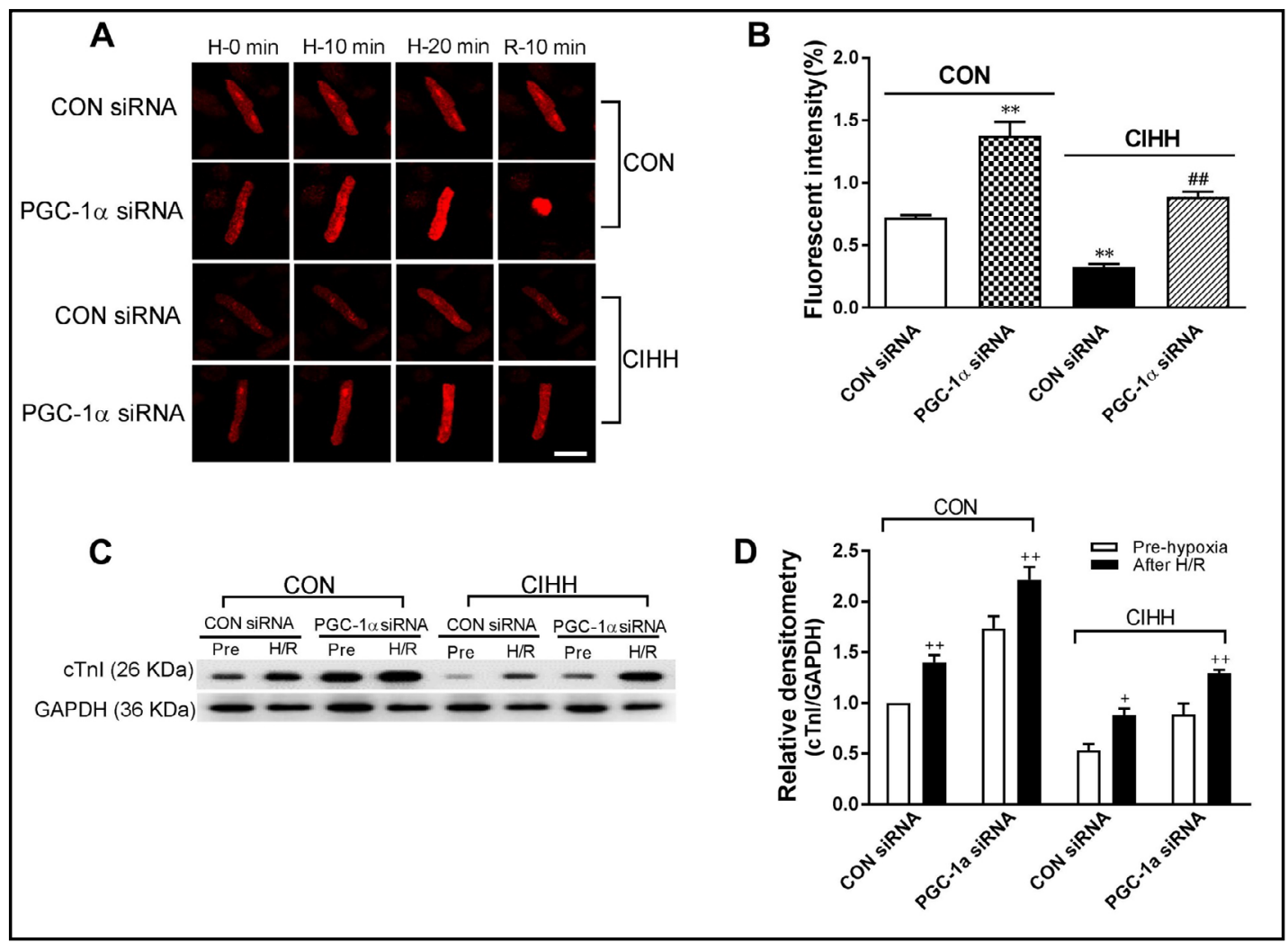

Fig. 3. PGC-1 $\alpha$ knocking down abrogates the protective effects of CIHH on cardiomyocytes. A: representative image shows changes of fluorescence images of $\left[\mathrm{Ca}^{2+}\right]_{\mathrm{i}}$ in single isolated rat cardiomyocytes before and during H/R by laser confocal microscopy. H-0 min: 0 min of hypoxia; H-10 min: 10 min of hypoxia; H-20 min: 20 min of hypoxia; R-10 min: $10 \mathrm{~min}$ of reoxygenation. Scale bar: $50 \mu \mathrm{m}$. B: changes of relative fluorescent intensity in single isolated rat cardiomyocytes of different groups after H/R. C and D: Representative Western blot images and summary data show the effect of CIHH on cTnI protein expression in cardiomyocytes before and after H/R. CON, control group; CIHH, CIHH group; Pre: pre-hypoxia; H/R: after 20-min hypoxia followed by 10 -min reoxygenation. Data are presented as mean $\pm \mathrm{SEM}, \mathrm{n}=6$ rats in each group. ${ }^{* *} \mathrm{p}<0.01 \mathrm{vs}$. CON siRNA of CON group; ${ }^{\# \#} \mathrm{p}<0.01$ vs. CON siRNA of CIHH group; ${ }^{+} \mathrm{p}<0.05,{ }^{++} \mathrm{p}<0.01$ vs. Pre-hypoxia.

\section{$P G C-1 \alpha$ siRNA adenovirus transfection inhibited the protein and $m R N A$ expression of PGC-}

$1 \alpha$

After cardiomyocytes were transfected with PGC-1 $\alpha$ siRNA adenovirus and cultured for 48 hours, rod-shaped cells with GFP fluorescence account for $70 \%$ of the total number of viable cardiomyocytes (Fig. 2A). Transfection of PGC- $1 \alpha$ siRNA significantly inhibited PGC$1 \alpha$ mRNA $(p<0.01$, Fig. 2 B) and protein $(p<0.01$, Fig. 2 C, D) expression in CON and CIHH myocytes compared with myocytes treated with control adenovirus.

\section{$P G C-1 \alpha$ is required for the protective effect of $\mathrm{CIHH}$ on cardiomyocytes}

The basal $\left[\mathrm{Ca}^{2+}\right]_{\mathrm{i}}$ in ventricular myocytes was similar in the CON and CIHH myocytes. After H/R, $\left[\mathrm{Ca}^{2+}\right]_{\mathrm{i}}$ increased in all groups of the myocytes. The increases of $\left[\mathrm{Ca}^{2+}\right]_{\mathrm{i}}$ in PGC$1 \alpha$ siRNA adenovirus-treated myocytes were much greater than control adenovirus-treated myocytes in both CON and CIHH myocytes ( $p<0.01$, Fig. 3A, B). Furthermore, PGC- $1 \alpha$ knockdown significantly increased the protein expression of $\mathrm{cTnI}$ before and after H/R both in CON and CIHH myocytes (Fig. 3C, D).These data suggest that PGC-1 $\alpha$ is required for the protective effect of CIHH on cardiomyocytes. 


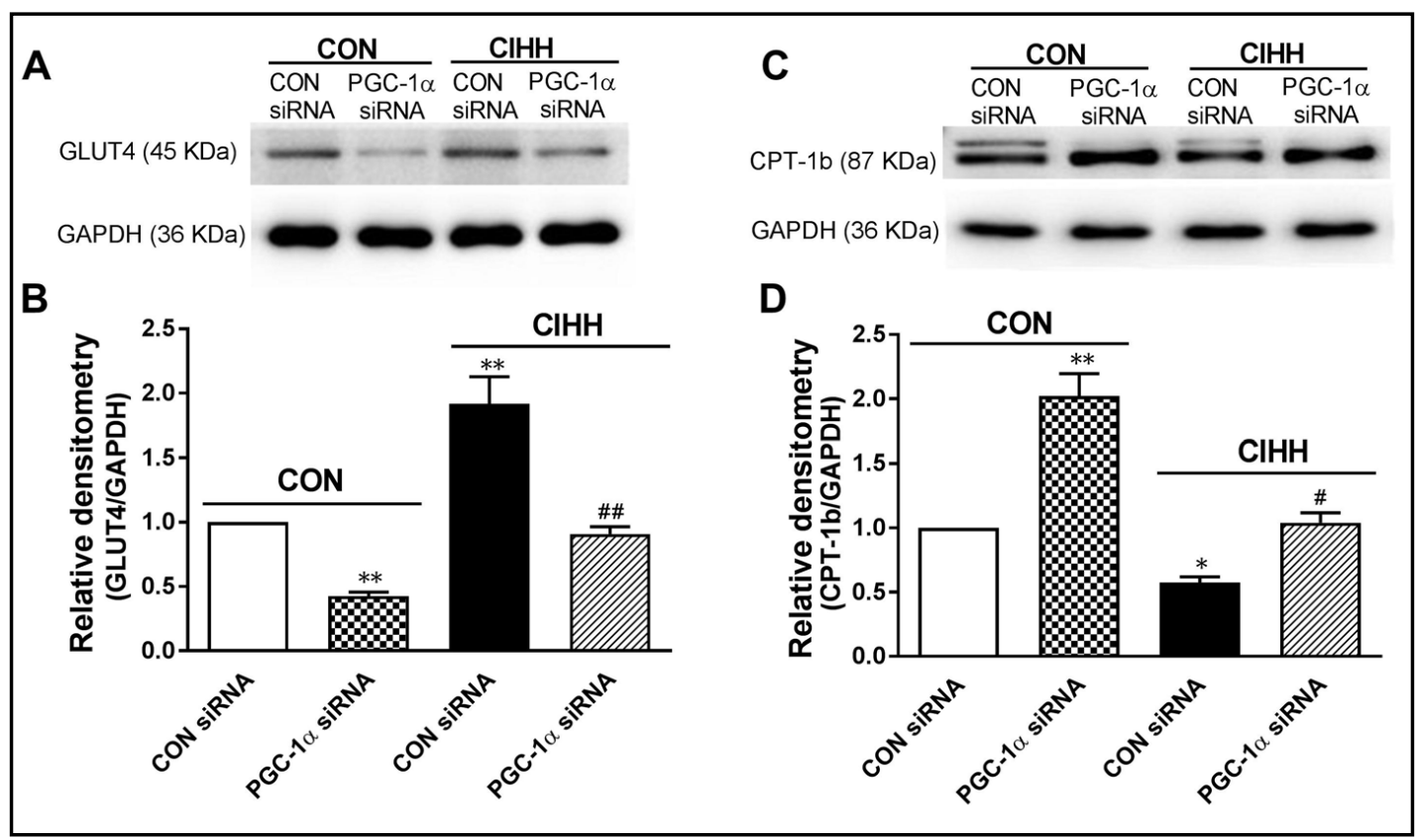

Fig. 4. GLUT4 and CPT-1b proteins expressions in cardiomyocytes after transfection with PGC-1 $\alpha$ siRNA adenovirus. Representative Western blot images and summary data shows changes of GLUT4 (A and B) and CPT-1b (C and D) proteins after transfection with PGC-1 $\alpha$ siRNA adenovirus. CON, control group; CIHH, CIHH group. Data are presented as mean \pm SEM, $n=6$ rats in each group. ${ }^{*} p<0.05,{ }^{* *} p<0.01$ vs. CON siRNA of CON group; ${ }^{\#} \mathrm{p}<0.05,{ }^{\# \#} \mathrm{p}<0.01$ vs. CON siRNA of CIHH group.

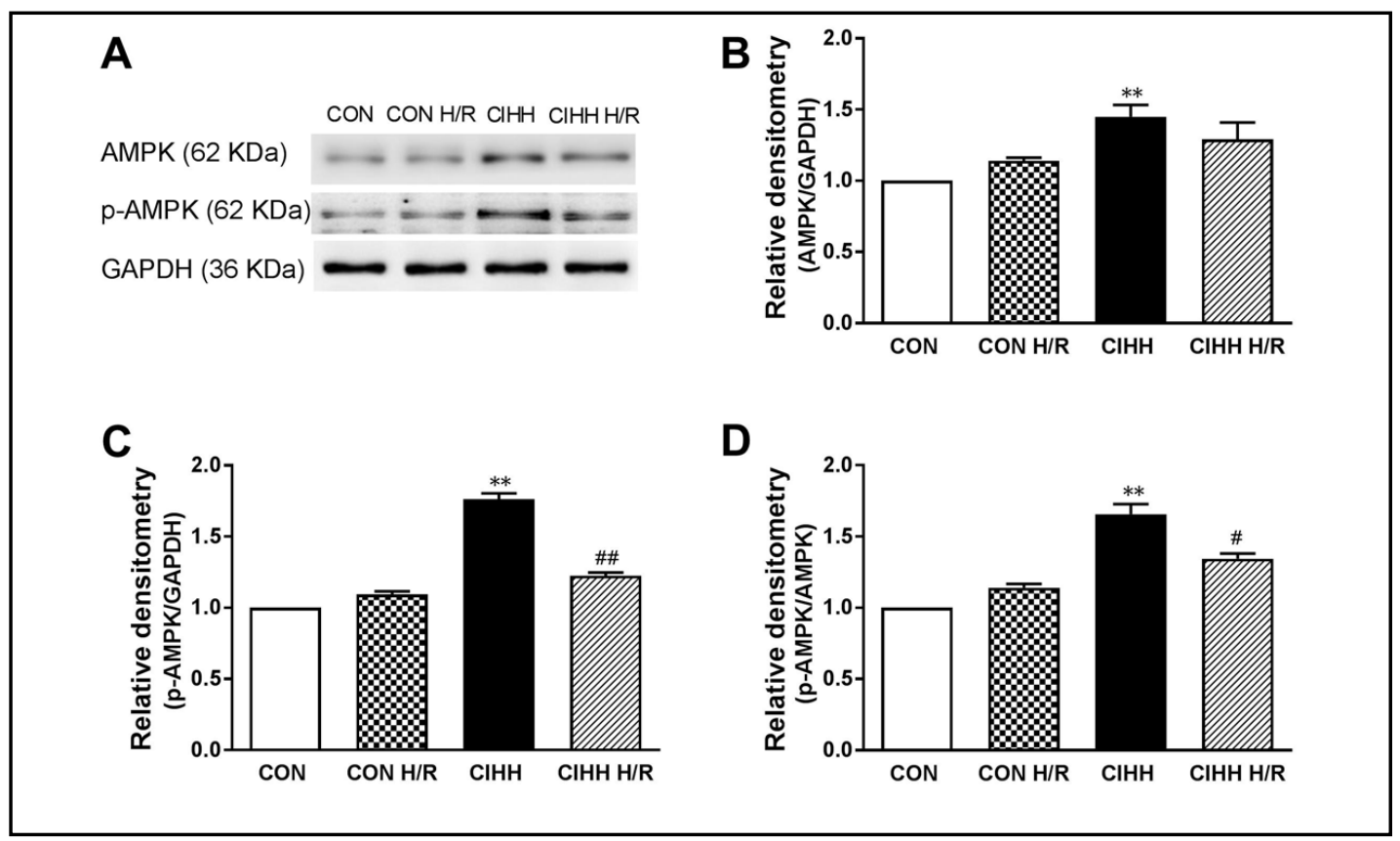

Fig. 5. CIHH treatment increases AMPK and p-AMPK proteins expressions in cardiomyocytes before and after H/R. Representative Western blot images (A) and summary data shows changes of AMPK (B), p-AMPK (C) protein expression, and p-AMPK/AMPK protein expression ratio (D) after CIHH treatment. CON, control group; CON H/R: control group with 20-min chemical hypoxia followed by 10-min reoxygenation; $\mathrm{CIHH}$, CIHH group; CIHH H/R, CIHH group with 20-min chemical hypoxia followed by 10-min reoxygenation. Data

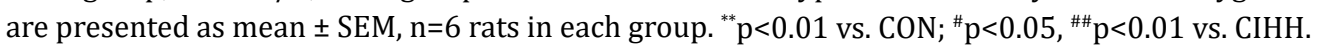




\section{Cellular Physiology Cell Physiol Biochem 2018;50:1891-1902 and Biochemistry \begin{tabular}{l|l} 
DOI: $10.1159 / 000494869$ & 2018 The Author(s). Published by S. Karger AG, Basel
\end{tabular}

Fig. 6. AMPK inhibitor dorsomorphin treatment reduces the protein expression of PGC- $1 \alpha$. Representative Western blot images (A) and summary data (B) shows PGC- $1 \alpha$ protein level in cardiomyocytes in the presence or absence (vehicle) of AMPK inhibitor dorsomorphin. CON, control group; $\mathrm{CIHH}, \mathrm{CIHH}$ group; Dors: dorsomorphin. Data are presented as mean \pm SEM, $n=6$ rats in each group. ${ }^{* *} \mathrm{p}<0.01$ vs. CON vehicle; ${ }^{\# \#} \mathrm{p}<0.01$ vs. CIHH vehicle.

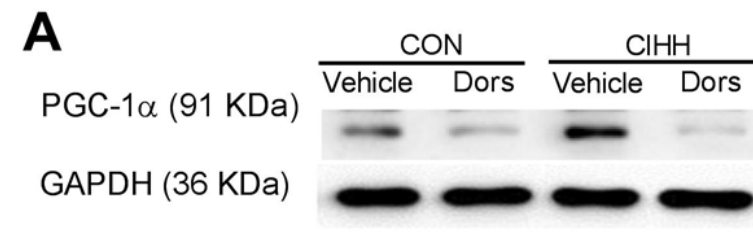

B

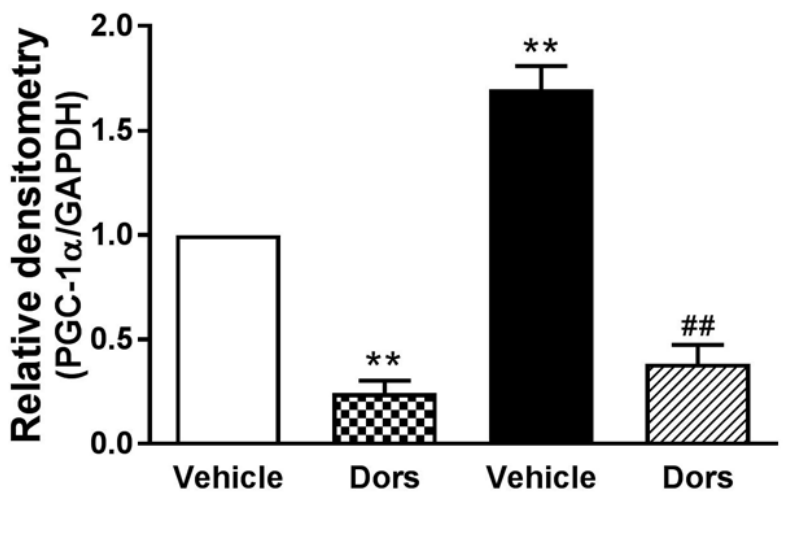

Effects of Down-regulation of PGC-1 $\alpha$ on GLUT4 and CPT-1b protein expressions in cardiomyocytes

After transfection of PGC-1 $\alpha$ siRNA adenovirus into cardiomyocytes, the expression of GLUT4 protein in CIHH cardiomyocytes was significantly inhibited ( $p<0.01$, Fig. $4 \mathrm{~A}, \mathrm{~B})$, while the expression of CPT- 1 b protein in cardiomyocytes was increased ( $p<0.05$, Fig. 4 C, D). In contrast, the expression of GLUT4 protein in CIHH cardiomyocytes was significantly increased $(p<0.01$, Fig. 4 A, B), while the expression of CPT- 1 b protein was decreased compared with that in CON cardiomyocytes after pretreated with control siRNA adenovirus ( $p<0.05$, Fig. 4 C, D). These findings indicate that CIHH could protect the cardiomyocytes against H/R injury through improving cardiac glucose and lipid metabolic disturbance via upregulation of PGC$1 \alpha$.

Phosphorylation of AMPK is critically involved in regulation of $P G C-1 \alpha$ pathway of CIHH

It is reported that chronic hypoxia could improve the glucose tolerance by activating the AMPK pathway in skeletal muscle [14]. Our study showed that AMPK, especially p-AMPK protein expressions were increased in cardiomyocytes after CIHH treatment, compared with CON cardiomyocytes ( $p<0.01$, Fig. 5). After treatment of cardiomyocytes with AMPK inhibitor dorsomorphin, $\mathrm{PGC}-1 \alpha$ protein expression was significantly decreased both in CON and $\mathrm{CIHH}$ cardiomyocytes ( $p<0.01$, Fig. 6). These results showed that phosphorylation of AMPK is critically involved in the regulation of PGC- $1 \alpha$ pathway of $\mathrm{CIHH}$.

\section{Effects of CIHH treatment on HIF-1 $\alpha$ protein expressions in cardiomyocytes}

Our study showed that $\mathrm{CIHH}$ treatment significantly increased the protein expression of HIF- $1 \alpha$ in cardiomyocytes under both baseline and H/R conditions compared with CON cardiomyocytes $(p<0.01$, Fig. 7$)$. This effect was consistent with elevated phosphorylation of AMPK. 


\section{Cellular Physiology Cell Physiol Biochem 2018;50:1891-1902

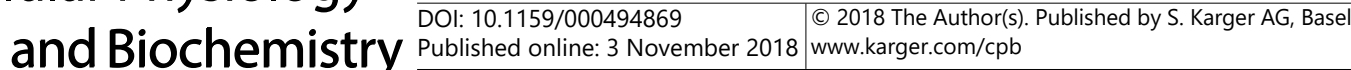 \\ Gu et al.: CIHH Protects Cardiomyocytes via PGC-1 $\alpha$}

Fig. 7. CIHH treatment increases HIF-1 $\alpha$ protein expression in cardiomyocytes before and after $H / R$. Representative Western blot images (A) and summary data (B) shows changes of HIF$1 \alpha$ protein level before and after H/R. CON, control group; CON H/R, control group with 20-min chemical hypoxia followed by 10-min reoxygenation; $\mathrm{CIHH}, \mathrm{CIHH}$ group; $\mathrm{CIHH} \mathrm{H} / \mathrm{R}$, CIHH group with 20-min hypoxia followed by 10-min reoxygenation. Data are presented as mean \pm SEM, $n=6$ rats in each group. ${ }^{* *} \mathrm{p}<0.01$ vs. $\mathrm{CON}$; ${ }^{*} \mathrm{p}<0.05$ vs. CIHH.

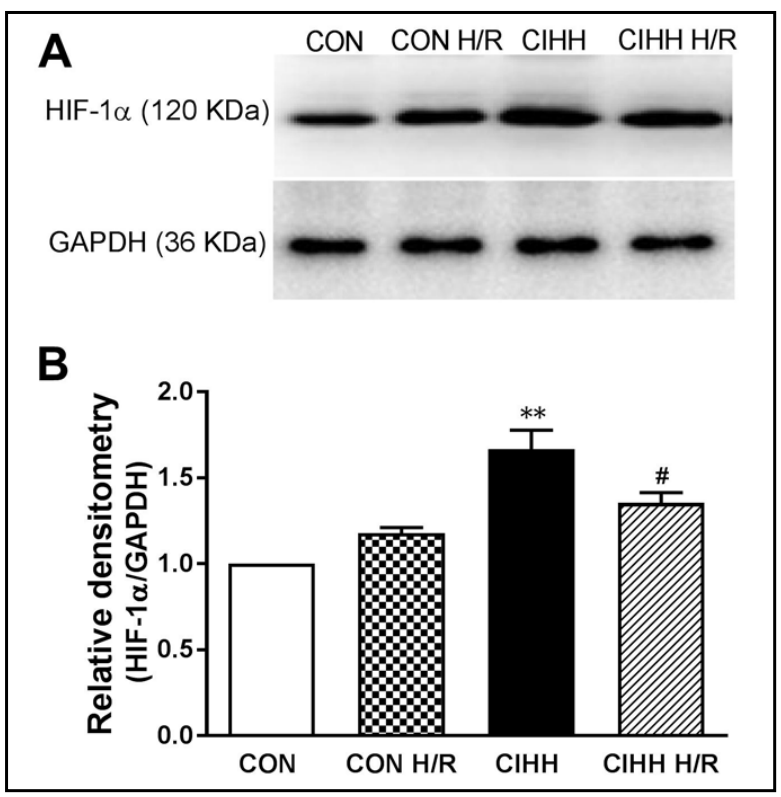

\section{Discussion}

During myocardial ischemia or hypoxia and early reperfusion or reoxygenation, intracellular $\left[\mathrm{Ca}^{2+}\right]_{i}$ increased dramatically, and consequent cardiac calcium overload aggravated the injury, edema and necrocytosis in cardiomyocytes. Intracellular free $\left[\mathrm{Ca}^{2+}\right]_{\mathrm{i}}$ abnormalities can also cause severe arrhythmia and ventricular fibrillation. Therefore, maintaining a relatively stable cytoplasmic $\left[\mathrm{Ca}^{2+}\right]_{\mathrm{i}}$ is significant for physiological activities of cardiomyocytes and cardiac function. $\mathrm{CIHH}$ treatment could reduce cardiac calcium overload and therefore enhance cardiomyocytes tolerance to I/R injury $[8,15,16]$. Our study further confirmed that $\mathrm{CIHH}$ treatment could reduce myocardial calcium overload and damage induced by $\mathrm{H} / \mathrm{R}$.

The normal structure and function of the heart depend on stablization of energy metabolism and disturbance of energy metabolism may lead to cardiac injury. There are a variety of factors involved in cardiac energy metabolism, in which peroxisome proliferators activated receptor-gamma coactivator-1 (PGC-1) is a well-known transcriptional regulator co-activator recognized in recent years. PGC- 1 family includes PGC- $1 \alpha$, PGC- $1 \beta$ and PRC (PGC-related coactivators). Among them, PGC-1 $\alpha$ plays an important role in the regulation of glucose and lipid metabolism through gene regulation [17]. Cardiac function was significantly inhibited after PGC-1 $\alpha$ gene knockdown [18], whereas increased PGC-1 $\alpha$ expression can enhance the tolerance of heart against ischemic injury [19]. Our recent study found that $\mathrm{CIHH}$ treatment could protect cardiac function by improving cardiac glucose metabolism through up-regulation of PGC- $1 \alpha$ [12]. In this study, when PGC- $1 \alpha$ of cardiomyocytes was knocked down by siRNA interference technology, the cardioprotective effect of CIHH was eliminated. It further confirmed that PGC- $1 \alpha$ was required for the protective effects of $\mathrm{CIHH}$ treatment.

PGC-1 $\alpha$ is a vital link in a regulatory network for metabolic homeostasis, which can further regulate the glucose uptake and fatty acids transport. Accumulation of fatty acid metabolites leads to cell acidosis, triggering overload of calcium ions, which in turn leads to myocardial systolic dysfunction and secondary membrane damage with increased sensitivity to I/R or $\mathrm{H} / \mathrm{R}$ injury [19-22], while improving the energy metabolism of the heart, such as increasing glucose metabolism in cardiomyocytes, can increase the tolerance of the heart to I/R or H/R injury [19, 20, 22]. GLUT4 has a high affinity for glucose molecules and is responsible for the uptake of glucose into cardiomyocytes [23]. While, carnitine palmitoyltransferase$1 \mathrm{~b}$ (CPT-1b), a channel enzyme located on the mitochondrial membrane, is responsible for 


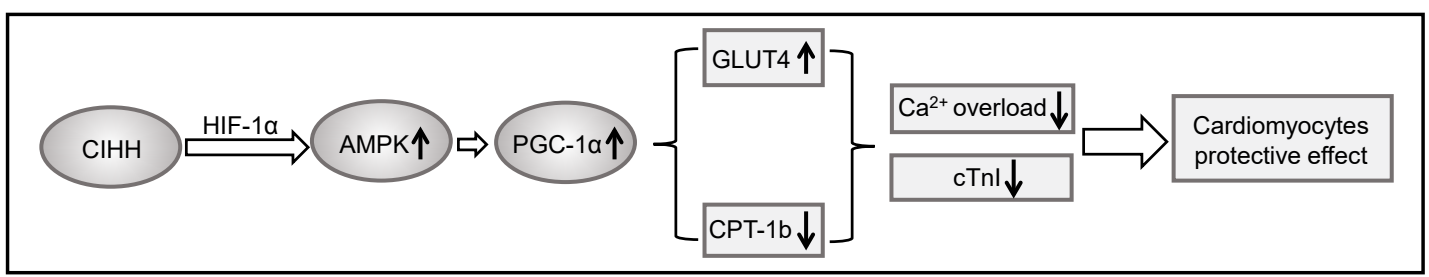

Fig. 8. Possible pathway shows the role of PGC-1 $\alpha$ in the protective effects of CIHH on cardiomyocytes against H/R injury.

transporting fatty acids into the mitochondria [24]. CPT-1b suppression has protective effect on cardiac morphology and function [25]. Therefore, the expression of GLUT4 and CPT-1b can well reflect the myocardial glucose and lipid metabolism. Studies have shown that PGC$1 \alpha$ could increase the tolerance of the heart to I/R or H/R injury by increasing the expression of glucose transporter 4 (GLUT4) [19, 22]. Consistent with those studies, we found that $\mathrm{CIHH}$ treatment protects cardiomyocytes with increased GLUT4 protein expression and reduced CPT-1b protein expression. After knocking down PGC-1 $\alpha$ using siRNA interference technology in the cardiomyocytes, the expression of GLUT4 protein decreased, while the expression of CPT-1b protein increased. Therefore, it confirmed that CIHH could optimize glucose and lipid metabolisms through regulating PGC- $1 \alpha$.

PGC- $1 \alpha$ activity can be finely tuned in response to different metabolic situations. Adenylate activated protein kinase (AMPK) can directly affect PGC- $1 \alpha$ activity through phosphorylation as a metabolic sensor. AMPK can regulate cell energy metabolism and organelle function by phosphorylating and activating PGC- $1 \alpha$ directly [26], and further regulating calcium ion in cardiomyocytes [27-29]. Recent studies have shown that an increased expression of AMPK, especially p-AMPK, plays an important role in protecting the heart against I/R or H/R injury [12, 22, 30-32]. In accordance with previous studies, our results indicated that CIHH treatment could increase the expression of AMPK protein, especially p-AMPK protein, in cardiomyocytes. Pretreatment with AMPK inhibitor dorsomorphin directly inhibited PGC$1 \alpha$ protein expression significantly in all groups. Collectively, our findings demonstrated that AMPK-PGC-1 $\alpha$ pathway was essential for the CIHH-induced cardioprotection.

HIF-1 is a key factor in hypoxia adaptation and regulates the expression of multiple hypoxic stress proteins [33]. HIF-1 $\alpha$ activates the decline of AMPK function caused by chronic kidney disease, confirming the close relationship between HIF-1 $\alpha$ and AMPK activation [34]. The expression of HIF-1 target genes and HIF-1-dependent receptors can also be reduced by pharmacologically blocking the AMPK activity [35]. HIF-1 also increases glycolysis, glucose metabolism and mitochondrial function to regulate energy metabolism [36, 37]. We showed that $\mathrm{CIHH}$ treatment significantly increased the expression of HIF- $1 \alpha$ in cardiomyocytes, which was consistent with the changes of AMPK and p-AMPK protein. Therefore, HIF-1 $\alpha-$ AMPK-PGC- $1 \alpha$ signaling pathway played an important role in CIHH-induced improvement of energy metabolism (Fig. 8).

\section{Conclusion}

In conclusion, this study provided substantial evidences that $\mathrm{CIHH}$ could reduce myocardial calcium overload and H/R injury through regulating glucose and lipid energy metabolism via HIF-1 $\alpha$-AMPK-PGC- $1 \alpha$ signaling pathway. Further studies are needed to determine whether PGC- $1 \alpha$ knocked down in vivo plays an important role in the cardioprotective effect of $\mathrm{CIHH}$, because the limitation of cardiomyocytes study cannot fully represent the whole heart function. This new information advances our understanding of the molecular mechanism involved in the protective effect of $\mathrm{CIHH}$, and suggests that targeting PGC- $1 \alpha$ might be used as a new strategy for treating cardiac ischemia or hypoxia diseases. 


\section{Cellular Physiology Cell Physiol Biochem 2018;50:1891-1902 and Biochemistry \begin{tabular}{l|l} 
DOI: $10.1159 / 000494869$ & \\
(c) 2018 The Author(s). Published by S. Karger AG, Basel \\
www.karger.com/cpb
\end{tabular}}

Gu et al.: $\mathrm{CIHH}$ Protects Cardiomyocytes via PGC- $1 \alpha$

\section{Acknowledgements}

This study was supported by the National Natural Sciences Foundation of China (No. 81200070, 31671184), the National Basic Research Development Program of China (No. 2012CB518200) and Natural Science Foundation of Hebei Province (H2015206512).

\section{Disclosure Statement}

The authors declare that no conflict of interests regarding this paper exist.

\section{References}

1 Sanchis-Gomar F, Perez-Quilis C, Leischik R, Lucia A: Epidemiology of coronary heart disease and acute coronary syndrome. Ann Transl Med 2016;4:256.

2 Kalogeris T, Baines CP, Krenz M, Korthuis RJ: Cell biology of ischemia/reperfusion injury. Int Rev Cell Mol Biol 2012;298:229-317.

-3 Zhang Y, Li XR, Zhao L, Duan GL, Xiao L, Chen HP: DJ-1 preserving mitochondrial complex I activity plays a critical role in resveratrol-mediated cardioprotection against hypoxia/reoxygenation-induced oxidative stress. Biomed Pharmacother 2018;98:545-552.

4 Anzawa R, Seki S, Horikoshi K, Taniguchi M, Mochizuki S: Exacerbation of acidosis during ischemia and reperfusion arrhythmia in hearts from type 2 Diabetic Otsuka Long-Evans Tokushima Fatty rats. Cardiovasc Diabetol 2007;6:17.

5 Harmancey R, Vasquez HG, Guthrie PH, Taegtmeyer H: Decreased long-chain fatty acid oxidation impairs postischemic recovery of the insulin-resistant rat heart. FASEB J 2013;27:3966-3978.

-6 Zhou JJ, Ma HJ, Liu Y, Guan Y, Maslov LN, Li DP, Zhang Y: The anti-arrhythmic effect of chronic intermittent hypobaric hypoxia in rats with metabolic syndrome induced with fructose. Can J Physiol Pharmacol 2015;93:227-232.

7 Zhang Y, Zhou ZN: Beneficial effects of intermittent hypobaric hypoxia on the body. Zhongguo Ying Yong Sheng Li Xue Za Zhi 2012;28:504-509.

-8 Ma HJ, Li Q, Ma HJ, Guan Y, Shi M, Yang J, Li DP, Zhang Y: Chronic intermittent hypobaric hypoxia ameliorates ischemia/reperfusion-induced calcium overload in heart via $\mathrm{Na} / \mathrm{Ca} 2+$ exchanger in developing rats. Cell Physiol Biochem 2014;34:313-324.

9 Bu HM, Yang CY, Wang ML, Ma HJ, Sun H, Zhang Y: K(ATP) channels and MPTP are involved in the cardioprotection bestowed by chronic intermittent hypobaric hypoxia in the developing rat. J Physiol Sci 2015;65:367-376.

10 Xie Y, Zhu WZ, Zhu Y, Chen L, Zhou ZN, Yang HT: Intermittent high altitude hypoxia protects the heart against lethal Ca2+ overload injury. Life Sci 2004;76:559-572.

11 Chen L, Lu XY, Li J, Fu JD, Zhou ZN, Yang HT: Intermittent hypoxia protects cardiomyocytes against ischemia-reperfusion injury-induced alterations in $\mathrm{Ca} 2+$ homeostasis and contraction via the sarcoplasmic reticulum and $\mathrm{Na}+\mathrm{Ca}$ + exchange mechanisms. Am J Physiol Cell Physiol 2006;290:C1221-1229.

12 Li X, Liu Y, Ma H, Guan Y, Cao Y, Tian Y, Zhang Y: Enhancement of Glucose Metabolism via PGC-1alpha Participates in the Cardioprotection of Chronic Intermittent Hypobaric Hypoxia. Front Physiol 2016;7:219.

13 Saito S, Furuno A, Sakurai J, Park HR, Shin-ya K, Tomida A: Compound C prevents the unfolded protein response during glucose deprivation through a mechanism independent of AMPK and BMP signaling. PLoS One 2012;7:e45845.

14 Yu L, Gong B, Duan W, Fan C, Zhang J, Li Z, Xue X, Xu Y, Meng D, Li B, Zhang M, Bin Z, Jin Z, Yu S, Yang Y, Wang $\mathrm{H}$ : Melatonin ameliorates myocardial ischemia/reperfusion injury in type 1 diabetic rats by preserving mitochondrial function: role of AMPK-PGC-1alpha-SIRT3 signaling. Sci Rep 2017;7:41337.

15 Zhang Y, Zhong N, Zhou ZN: Effects of chronic intermittent hypobaric hypoxia on the L-type calcium current in rat ventricular myocytes. High Alt Med Biol 2010;11:61-67.

16 Ding HL, Zhu HF, Dong JW, Zhu WZ, Zhou ZN: Intermittent hypoxia protects the rat heart against ischemia/ reperfusion injury by activating protein kinase C. Life Sci 2004;75:2587-2603. 


\section{Cellular Physiology Cell Physiol Biochem 2018;50:1891-1902 \begin{tabular}{ll|l|l|l|l} 
DOI: $10.1159 / 000494869$ & 2018 The Author(s). Published by S. Karger AG, Basel \\
and Biochemistry & Published online: 3 November 2018 & www.karger.com/cpb
\end{tabular}}

Gu et al.: $\mathrm{ClHH}$ Protects Cardiomyocytes via PGC- $1 \alpha$

17 Huss JM, Kelly DP: Nuclear receptor signaling and cardiac energetics. Circ Res 2004;95:568-578.

18 Mootha VK, Handschin C, Arlow D, Xie X, St Pierre J, Sihag S, Yang W, Altshuler D, Puigserver P, Patterson N, Willy PJ, Schulman IG, Heyman RA, Lander ES, Spiegelman BM: Erralpha and Gabpa/b specify PGC-1alphadependent oxidative phosphorylation gene expression that is altered in diabetic muscle. Proc Natl Acad Sci U S A 2004;101:6570-6575.

19 Rowe GC, Jiang A, Arany Z: PGC-1 coactivators in cardiac development and disease. Circ Res 2010;107:825838.

-20 Finck BN, Kelly DP: Peroxisome proliferator-activated receptor gamma coactivator-1 (PGC-1) regulatory cascade in cardiac physiology and disease. Circulation 2007;115:2540-2548.

-21 Li YJ, Wang PH, Chen C, Zou MH, Wang DW: Improvement of mechanical heart function by trimetazidine in db/db mice. Acta Pharmacol Sin 2010;31:560-569.

-22 Kuznetsov AV, Javadov S, Sickinger S, Frotschnig S, Grimm M: H9c2 and HL-1 cells demonstrate distinct features of energy metabolism, mitochondrial function and sensitivity to hypoxia-reoxygenation. Biochim Biophys Acta 2015;1853:276-284.

-23 Nagendran J, Pulinilkunnil T, Kienesberger PC, Sung MM, Fung D, Febbraio M, Dyck JR: Cardiomyocytespecific ablation of CD36 improves post-ischemic functional recovery. J Mol Cell Cardiol 2013;63:180-188.

24 Kolwicz SC, Jr., Purohit S, Tian R: Cardiac metabolism and its interactions with contraction, growth, and survival of cardiomyocytes. Circ Res 2013;113:603-616.

25 Zhang Y, Fang X, Dai M, Cao Q, Tan T, He W, Huang Y, Chu L, Bao M: Cardiac-specific down-regulation of carnitine palmitoyltransferase-1b (CPT-1b) prevents cardiac remodeling in obese mice. Obesity (Silver Spring) 2016;24:2533-2543.

26 Jager S, Handschin C, St-Pierre J, Spiegelman BM: AMP-activated protein kinase (AMPK) action in skeletal muscle via direct phosphorylation of PGC-1alpha. Proc Natl Acad Sci U S A 2007;104:12017-12022.

-27 Canto C, Gerhart-Hines Z, Feige JN, Lagouge M, Noriega L, Milne JC, Elliott PJ, Puigserver P, Auwerx J: AMPK regulates energy expenditure by modulating NAD+ metabolism and SIRT1 activity. Nature 2009;458:10561060 .

28 Scarpulla RC: Metabolic control of mitochondrial biogenesis through the PGC-1 family regulatory network. Biochim Biophys Acta 2011;1813:1269-1278.

-29 Iwabu M, Yamauchi T, Okada-Iwabu M, Sato K, Nakagawa T, Funata M, Yamaguchi M, Namiki S, Nakayama R, Tabata M, Ogata H, Kubota N, Takamoto I, Hayashi YK, Yamauchi N, Waki H, Fukayama M, Nishino I, Tokuyama K, Ueki K et al.: Adiponectin and AdipoR1 regulate PGC-1alpha and mitochondria by $\mathrm{Ca}(2+)$ and AMPK/SIRT1. Nature 2010;464:1313-1319.

-30 Gundewar S, Calvert JW, Jha S, Toedt-Pingel I, Ji SY, Nunez D, Ramachandran A, Anaya-Cisneros M, Tian R, Lefer DJ: Activation of AMP-activated protein kinase by metformin improves left ventricular function and survival in heart failure. Circ Res 2009;104:403-411.

-31 Gauthier MS, O’Brien EL, Bigornia S, Mott M, Cacicedo JM, Xu XJ, Gokce N, Apovian C, Ruderman N: Decreased AMP-activated protein kinase activity is associated with increased inflammation in visceral adipose tissue and with whole-body insulin resistance in morbidly obese humans. Biochem Biophys Res Commun 2011;404:382-387.

-32 Kim M, Tian R: Targeting AMPK for cardiac protection: opportunities and challenges. J Mol Cell Cardiol 2011;51:548-553.

33 Malec V, Gottschald OR, Li S, Rose F, Seeger W, Hanze J: HIF-1 alpha signaling is augmented during intermittent hypoxia by induction of the Nrf2 pathway in NOX1-expressing adenocarcinoma A549 cells. Free Radic Biol Med 2010;48:1626-1635.

-34 Li H, Satriano J, Thomas JL, Miyamoto S, Sharma K, Pastor-Soler NM, Hallows KR, Singh P: Interactions between HIF-1alpha and AMPK in the regulation of cellular hypoxia adaptation in chronic kidney disease. Am J Physiol Renal Physiol 2015;309:F414-428.

-35 Lee M, Hwang JT, Lee HJ, Jung SN, Kang I, Chi SG, Kim SS, Ha J: AMP-activated protein kinase activity is critical for hypoxia-inducible factor- 1 transcriptional activity and its target gene expression under hypoxic conditions in DU145 cells. J Biol Chem 2003;278:39653-39661.

-36 Tekin D, Dursun AD, Xi L: Hypoxia inducible factor 1 (HIF-1) and cardioprotection. Acta Pharmacol Sin 2010;31:1085-1094.

-37 Loor G, Schumacker PT: Role of hypoxia-inducible factor in cell survival during myocardial ischemiareperfusion. Cell Death Differ 2008;15:686-690. 